\title{
IR Thermography for Quality Assessment of Microelectronic Devices
}

\author{
Z. Radivojevic ${ }^{1}$, I. Kassamakov ${ }^{2,3}$, M. Oinonen ${ }^{3,4}$, P. Vihinen ${ }^{2}$, H. Seppanen ${ }^{2,3}$ \\ ${ }^{1}$ Nokia Research Center, P.O.Box 407, FIN-00045, Helsinki, Finland \\ ${ }^{2}$ Department of Physical Sciences, P.O.Box 64, FIN-00014, Univ. of Helsinki, Finland \\ ${ }^{3}$ Helsinki Institute of Physics, P.O.Box 64, FIN-00014, University of Helsinki, Finland \\ ${ }^{4}$ Detector Laboratory, P.O.Box 64, FIN-00014, University of Helsinki, Finland
}

\begin{abstract}
This work presents collaboration between electronics industry and University aimed to develop novel power cycling methodology combined with IR thermography for quality and reliability studies of microelectronic assemblies. For this purpose an array of programmable Thermo Electric Converters (TEC) was designed and constructed [1]. The array provides possibility of introduction of controlled anisothermal temperature field as a function of time. Such field is exploited for stimulation for IR thermography and lifetime acceleration experiments. Furthermore, an advanced method, which takes advantage of short stimulations by photon blast was used to perform Time Domain IR Thermography. Combination of such techniques provided information about the weakest points in the assembly, design and optimisation of the manufacturing processes.
\end{abstract}

\section{Introduction}

Both the industrial and scientific reasons lead to smaller object sizes in microelectronics. Electronics industry continues to demand higher power while reducing the size of an electronics enclosure. With miniaturization and mass reduction the power dissipation density increases rapidly challenging long-term reliability entangled by thermally driven effects. The reliability is very important especially for objects containing different materials with different properties such as thermal expansion characteristics etc. Popularity of the thermal cycling test (isothermal air to air heating) is at least in part owed to the simplicity of concept and the simplicity of operation. However, in recent years, there is a growing debate on the validity of thermal cycling tests since under the real field of usage the heat is generated by a powered electronic device and as a result the assembly is under anisothermal conditions. Furthermore, as the whole thermal mass of an electronic enclosure gets smaller, failure root cause becomes dominated by anisothermal effects [2]. These reasons represent basic needs for introduction of transient anisothermal temperature field (ATF) into quality assessment procedure.

In this work a device and a method providing ATF was designed and constructed. Such ATF was combined with IR thermography on several microelectronics assemblies. For very low thermal mass systems like Chip-on-Flex (CoF) with large surface-to-volume $(S / V)$ ratio an advanced stimulation by intensive light pulses (photon blast from powerful flash) was used to perform Time-Domain IR Thermography (TDIRT). These pulses induced stimulation and propagation of thermal wave across the assembly. The heat wave propagation is a function of interconnection structures and materials involved. Defects in such an assembly (induced by thermomechanical stresses or poor interfacial contact areas) can be recognised by tracing pattern of transient properties of the heat flow. Different phenomena are developing at interconnection interfaces induced by "inner-life" of an assembly. For example, electrical interconnections are mainly based on intermetallic layer structures formed over certain areas. When transient anisothermal field is 
introduced across the area the heat path is influenced by defects created as a result of experienced stresses and/or poor manufacturing process. Such physical features were exploited in TDIRT technique to locate and identify risky areas in microelectronic assemblies. With nanosecond time stimulation (photon blast), micrometer-scale resolution was obtained. Effects of different material interfacial conductance and defects were obtained. For larger assemblies like Ball Grid Array (BGA) packages with low S/V ratio, the ATF field was provided by TEC modules for IR sequential imaging. The TEC provided programmable temperature operating conditions. By this combination it was possible to distinguish in homogeneities in the heat flow and recognise high risk regions for possible failures. In addition, defects were also recognised by comparison of transient temperature curves above good and poor areas where a decrease in thermal conductivity was observed.

\section{Experimental technique and results}

The ATF field for stimulation of different devices under test (DUT) was provided either by using photon blast or by programmable TEC modules. For IR thermography, we have used a FLIR SC3000 Quantum Well IR Photo-detector (QWIP) camera equipped with three lenses, operating at a wavelength of 8-9 $\mu \mathrm{m}$. Prior the IR measurements the DUTs were coated with special black paint in order to obtain high and uniform emissivity and to prevent radiation reflections from the ambient.

\subsection{TEC programmable array}

Multipurpose programmable TEC array was designed and constructed for lifetime acceleration, IR thermography and material property tests. The system consists of 12 independent channels each capable of providing transient T-field with adjustable rate of change in range of $0^{\circ}<\mathrm{dT} / \mathrm{dt}<50^{\circ} \mathrm{C} / \mathrm{min}$ and T-extremes as; $T_{\min }=-40^{\circ} \mathrm{C}$ and $T_{\max }=+150^{\circ} \mathrm{C}$. Functional scheme and typical T-profile provided by a single TEC are presented in Fig $1 \mathrm{a}, \mathrm{b})$. The ATF field was used for stimulation across assemblies with BGA packages. The IR imaging was taken across the assembly showing thermal gradients introduced into the tests (see Fig $2 \mathrm{~b}$ ). Relatively good resolution was achieved capable to provide information about thermal gradients at a single solder joint level. This information was further exploited for validation of simulation models for temperature distribution and stress analysis. 


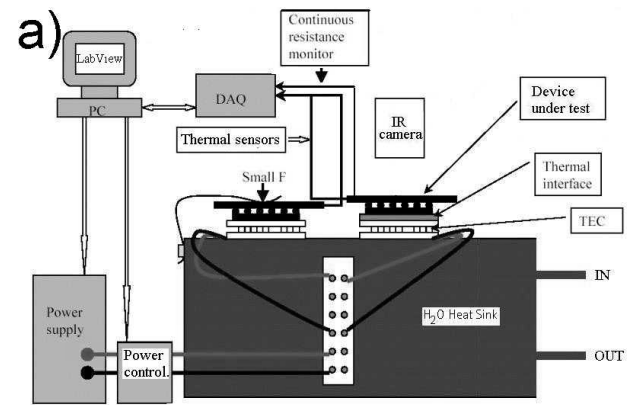

Figure 1. a) Functional scheme of TEC array. b)

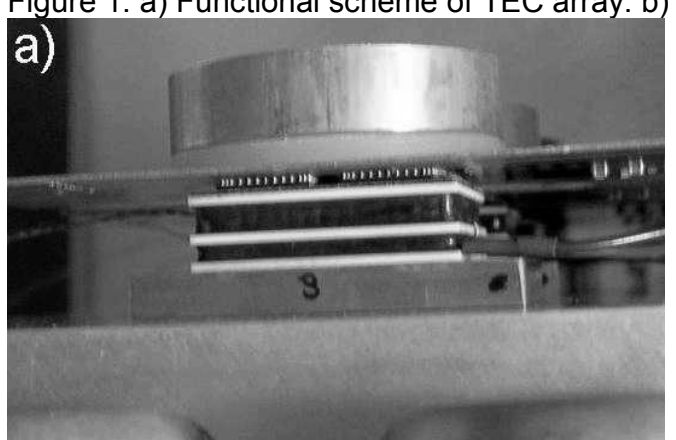

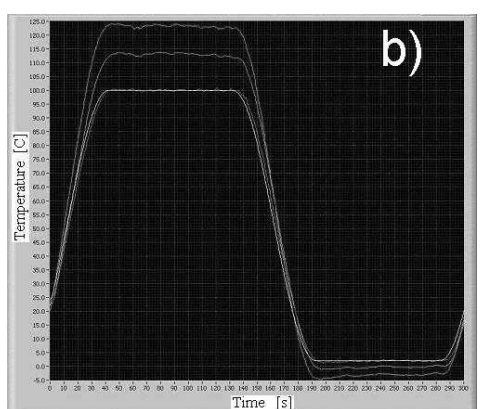

Usual temperature profile.

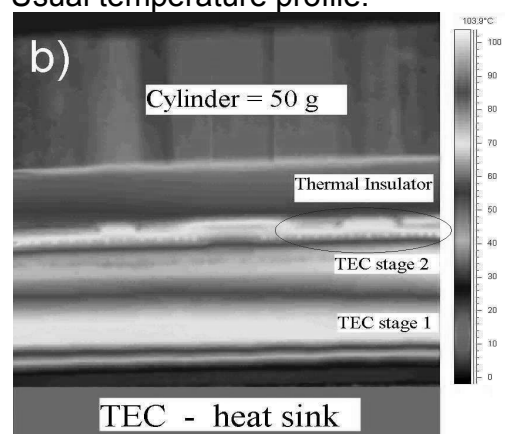

Figure 2. a) TEC module with BGA assembly attached upside-down. b) IR image taken across the assembly showing anisothermal temperature field across the BGA. Notice small structures related to solder joint interconnections $(\varnothing \sim 0.3 \mathrm{~mm})$.

\subsection{Stimulation by photon blast}

Chip-on-Flex (CoF) assembly with $11 \times 4 \times 0.15 \mathrm{~mm}^{3}$ die (samples courtesy of ALICE collaboration [3]) was single-point TAB-bonded to ultra-light flexible aluminium-polyimide cable. Taking advantage of very low thermal mass and large $S / V$ ratio the CoF assembly was investigated by TDIRT technique combined with short photon blast stimulation. The photon blast consisted of $10 \mathrm{~ns}$ long light pulse from 1.5 $\mathrm{kW}$ powerful flash (see Fig 3a). The CoF assembly was attached via the die to the cooled plate (see Fig $3 \mathrm{~b}$ ) to lower temperature to $15^{\circ} \mathrm{C}$ and increase the IR resolution. Structures on the CoF involving very thin Al traces (width $36 \mu \mathrm{m}$, pitch $80 \mu \mathrm{m}$, thickness $14 \mu \mathrm{m}$ ) are shown in Fig 4a. To test and calibrate the TDIRT technique calibration CoF sample with intentionally made failures was made. Every $6^{\text {th }}$ line was left non-connected (see Fig 4b) providing a good testing pattern. Furthermore, a case with intentionally missed two neighbouring lines was made to challenge and check resolution of the TDIRT technique. A good quality bond forms a thermal contact to the cooled die showing up as a colder interconnection compared to a non-connected one. The calibration CoF was further stimulated by photon blast and TDIRT was performed. Results are presented in Fig 5. Materials involved in the CoF construction result in the heat diffusion length $(\sqrt{D t})$ of about $1 \mu \mathrm{m}$. The variable $D=k / \rho C$ thermal diffusivity is related to $k$ - thermal conductivity, $\rho$ - the density and $C$ - the specific heat. Those parameters are subjected to change as a function of loading and assembling conditions [4]. Combination of rapid stimulation and adequate IR imaging yielded in sufficient resolution to distinguish defects at a single interconnection level. The best resolution was achieved at cooling phase right after the stimulation (marked as $\mathrm{t}=0$ ) as shown in Fig 5 . 
http://dx.doi.org/10.21611/qirt.2004.087

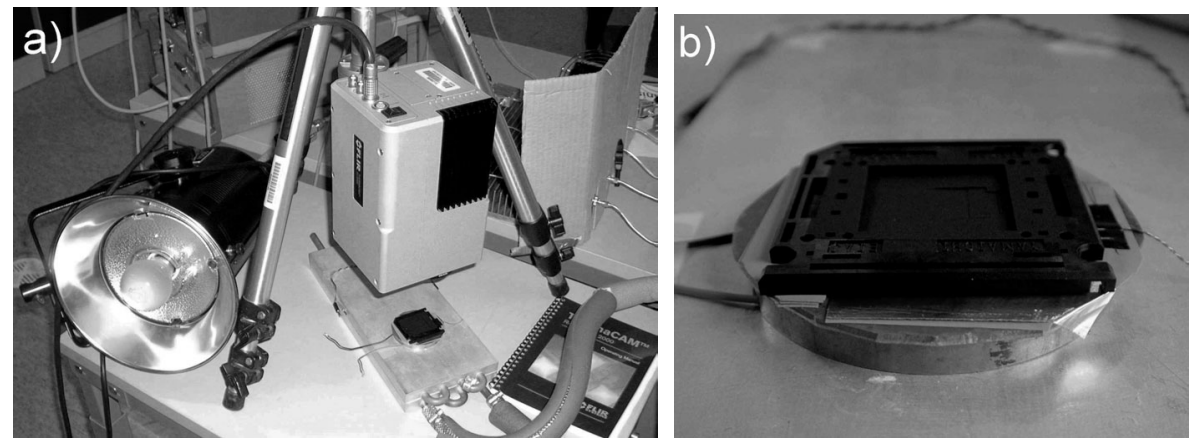

Figure 3. a) TDIRT set-up. b) Painted CoF sample and cooling jig.

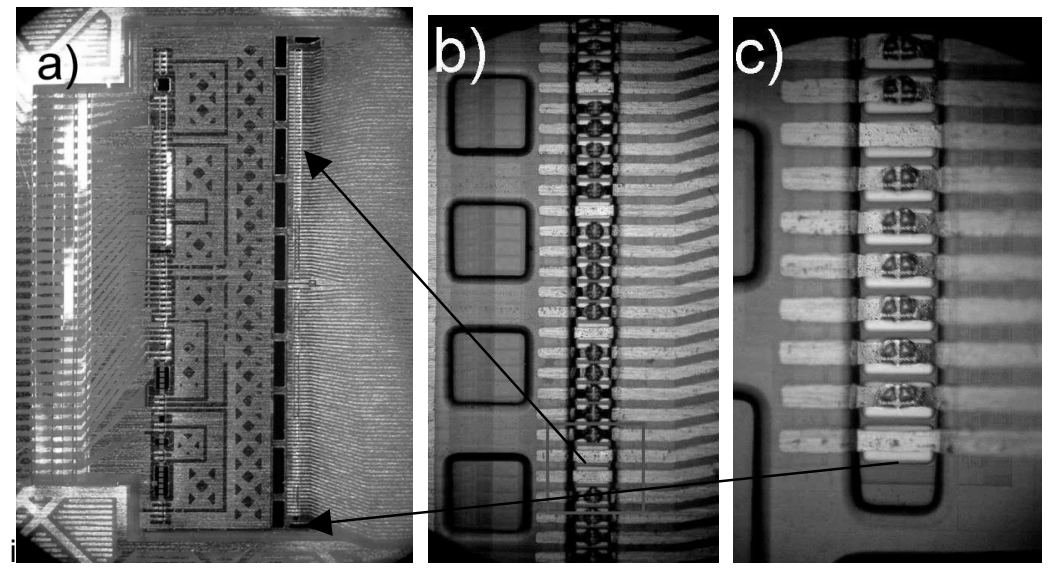

Figure 4. a) Structure of Chip-on-Flex assembly and intentionally made for calibration purpose. b) Notice intentionally missed every $6^{\text {th }}$ bond and case with two missed neighbouring bonds.

Obtained resolution was enough to distinguish even two neighbouring non-connected bonds. The sets of 5 good bonds show up as "cold spots" separated by a bad bond (see Fig 5 at $\mathrm{t}=0$ ). 

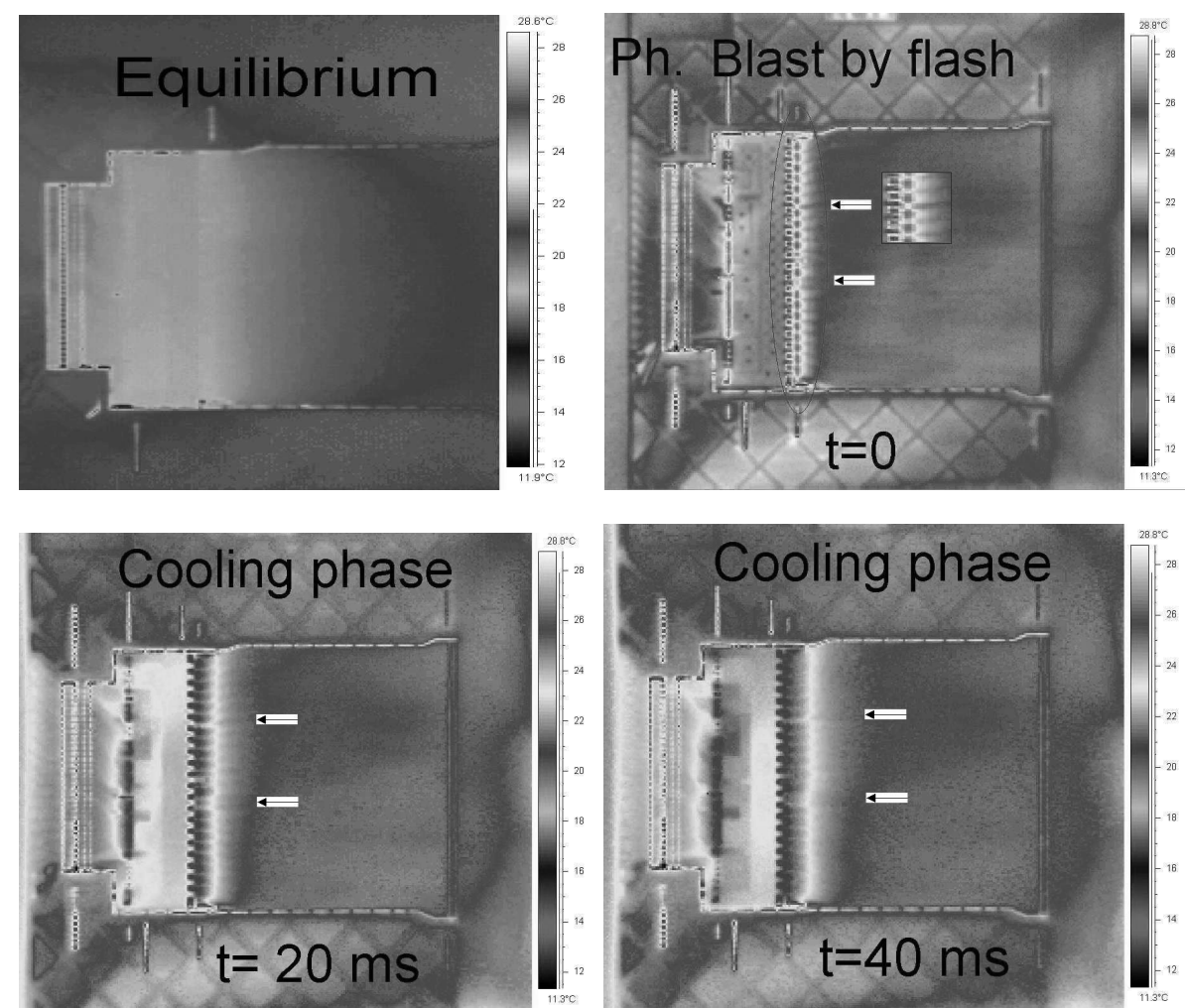

Figure 5. Results of TDIRT measurements with calibration CoF sample. Notice distinguishable defect caused by intentionally missed two neighbouring bonds (zoomed square at $\mathrm{t}=0 \mathrm{~ms}$ ).
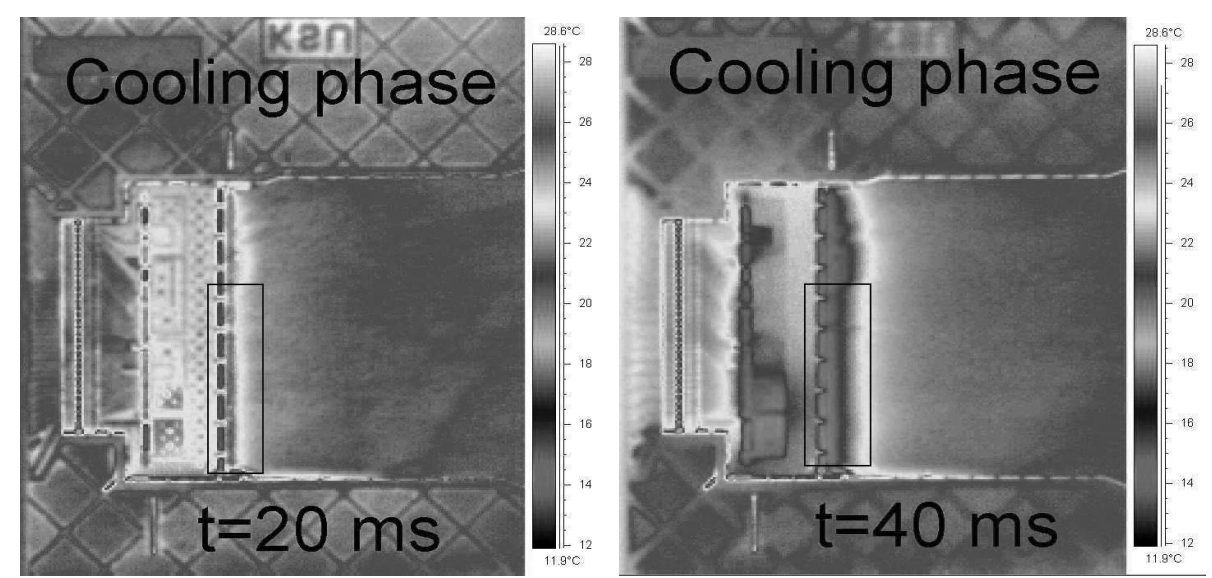

Figure 6. Results of TDIRT measurements on "real" CoF sample with accidental defects caused by shifting of the manufacturing parameters (marked by black square). 
Once calibrated and checked the TDIRT technique was applied to "real" samples containing failures created accidentally (by shifting of the manufacturing parameters out of optimal window). Results are shown in Fig 6 where is possible to distinguish different regions in thermal pattern attributed to differences in heat flow and quality of interconnects. Notice hot regions in the pattern where the heat front propagation separates good and poor areas. This information is very important when optimising assembling parameters and performing overall quality inspection.

\subsection{Stimulation by TEC array}

The CoF was attached upside-down via the die to the TEC which provided the ATF field for IR sequential imaging. Loading profile consisted of $3 \mathrm{~min}$ ramp-up/down and 4 min steady state temperatures at extremes of $T_{\min }=2^{\circ} \mathrm{C}$ and $T_{\max }=100^{\circ} \mathrm{C}$. Results are presented in Fig 7 . The highest resolution was achieved at $90^{\circ} \mathrm{C}$ working temperature where it was possible to distinguish regions with different quality of the interconnections (seen Fig 7b). Furthermore, off-line analysis allowed selection of regions by comparison of temperature transient curves attributed to different areas. Result of such analysis is given in Fig 7c). Notice difference in transient behaviour and a decrease in thermal conductance attributed to poor interconnection regions.
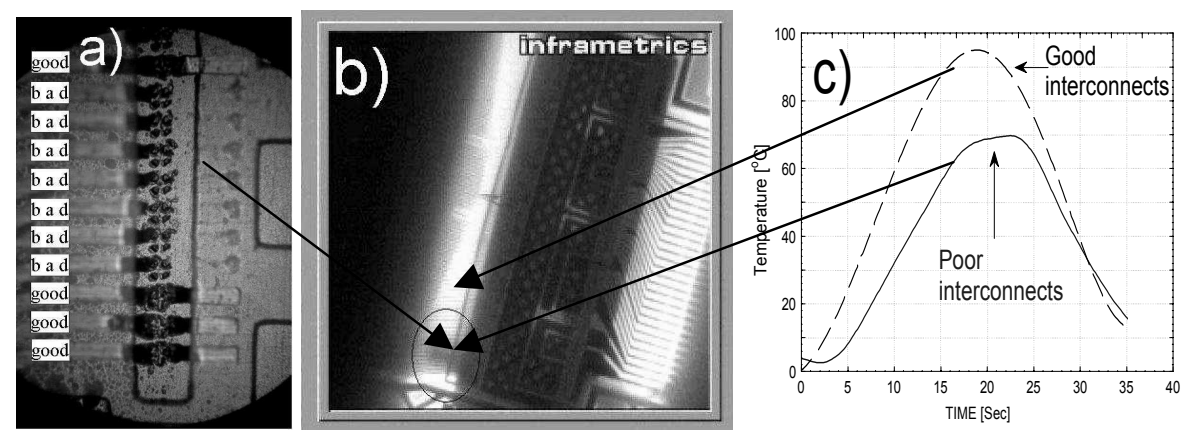

Figure 7. Sequential IR imaging. a) CoF sample with 7 poor interconnection lines. b) IR temperature map with marked region related to poor interconnects. c) Observed decrease in thermal conductance above poor interconnections. 


\section{Discussion and conclusion}

In this work IR thermography was combined with different techniques for transient anisothermal stimulations of several microelectronic assemblies. Low thermal mass systems with large S/N ration (like CoF) present very suitable assembly to be investigated by TDIRT technique combined with photon blast stimulation. In this case very fast method based on TDIRT technique for qualification of interconnections was described. Achieved high resolution $(<80 \mu \mathrm{m})$ was capable of distinguishing assembling defects across two neighbouring traces into the CoF. Such technique presents relatively simple, fast and easy method to recognise defects caused by not optimised assembling conditions. If combined with removable materials for uniform emissivity on the surface it could present good technique for very fast selection of good samples from the bad ones in a production line.

In case of using the TEC for stimulation good resolution was achieve at steady state temperatures of about $90^{\circ} \mathrm{C}$. With this conditions sequential IR imaging was capable of distinguishing risky regions by using transient temperature loading and comparison of extracted transient T-curves above good and poor interconnections. When certain defect is grown at the interfacial layer it is reflected as an additional decrease of thermal conductance across such area. These physical properties yielded in possibilities of identification of failure risky interconnections.

In case of relatively larger thermal masses and S/V ratio (like BGA assemblies) the TEC modules were used to provide ATF stimulation. IR measurements yielded in relatively sufficient resolution to resolve small thermal gradients even at the level of a single solder joint. This information is very important for validation and refining of thermal and stress simulation models.

As a final conclusion we would like to emphasize extremely useful technique based on IR thermography for quality assessment of microelectronic assemblies, which can provide fast quality inspection and feedback to design and optimisation line.

\section{REFERENCES}

[1] Vihinen P., Thermoelectric cooler array for lifetime studies of microelectronic devices, Proceeding of The XXXVIII Annual Conference of the Finnish Physical Society, Oulu, 18.-20.3.2004. p. 79.

[2] Pecht M.G., Influence of Temperature on Microelectronics and System Reliability, CRC Press 1997.

[3] http://alice.web.cern.ch/Alice/AliceNew/

[4] D.G.Cahill et al, Nanoscale thermal transport, J.Appl.Phys, 93(2):793818, 2003. 